\title{
Synthesis and Characterization of Polystyrene Grafted Nanohybrids by Graft Polymerization
}

\author{
Shaista Taimur ${ }^{1,2}$, Tariq Yasin $^{1}$, Saira Bibi ${ }^{1}$ \\ ${ }^{1}$ Department of Metallurgy and Materials Engineering, Pakistan Institute of Engineering and Applied Sciences, PO Nilore, \\ Islamabad, Pakistan \\ shaista.taimur@buitms.edu.pk; tariqyasin@pieas.edu.pk; sairabushi@gmail.com \\ ${ }^{2}$ Faculty of Engineering, Balochistan University of Information Technology, Engineering and Management Sciences, \\ Baleli, Quetta, Pakistan
}

\begin{abstract}
Novel polystyrene grafted sepiolite nanohybrid material (SPS) has been synthesized by using two different but greener routes i.e. simultaneous radiation grafting (bulk grafting) and emulsion graft polymerization. In case of simultaneous radiation grafting, influence of absorbed dose and monomer concentration were studied whereas for emulsion graft polymerization, outcome of monomer quantity was investigated. The grafting yield (GY) was measured by gravimetric analysis. The grafting of polystyrene chains was verified by Fourier transform infrared spectroscopy (FT-IR). The structural and morphological studies of synthesized nanohybrids have been explored by X ray diffraction (XRD) and field emission-scanning electron microscopy (FESEM) respectively. The grafting yield by simultaneous radiation grafting and emulsion graft polymerization was found to be $257 \%$ and $85 \%$ respectively. Transmission electron microscope (TEM) images evidently revealed the shortening of sepiolite nanofibers after silanization due to which heterogeneous nucleation occurred in micelles in emulsion graft polymerization. Results showed that simultaneous radiation grafting was comparatively better technique for grafting polystyrene chains onto silanized sepiolite.
\end{abstract}

Keywords: Sepiolite, Styrene, Nanohybrids, Simultaneous Radiation Grafting, Emulsion Graft Polymerization.

\section{Introduction}

Polymer modification by grafting of monomers into some substrate is aimed to develop restructured materials having certain properties for pragmatic applications. Radiation-induced graft polymerization is a deep-rooted knowledge about developing grafted polymers [1-3]. The simultaneous radiation grafting is a one-step modus operandi in which free radicals are generated equivalently on monomer and substrate causing higher grafting efficiency. Bulk grafting by radiation processing is a simple, clean and environmentally friendly method as there is no need of solvents, initiators or high temperatures, leading to the development of tailored materials.

Likewise, emulsion grafting is a greener practise as it is involved with the consumption of water as solvent which makes this method eco-friendly. It permits significant heat dissipation during the polymerization process. Emulsion polymerization is an elaborated heterogeneous system which prospers through free radical mechanism. A surfactant is used to emulsify a comparatively hydrophobic monomer and water while an initiator is required to generate free radicals and polymerization reaction results in the development of a latex [4]. Emulsion polymerization technique is used by many researchers for grafting purpose $[5,6]$.

Recently, organic-inorganic nanohybrids obtained via different synthesis routes have emerged into potent substitutes to traditional polymer composites in both academic and industrial arenas. As a minimum requirement, nanohybrids have one distinctive dimension in nanometer scale [7-11]. Clays are used as inorganic component of the nanohybrids to develop clay polymer nanocomposites (CPNs). Clays are considered as low-cost, non-hazardous and ubiquitous nanoscale materials [12]. CPNs have come to light as elegantly designed materials due to their significant properties and widespread applications in almost every field [13-15]. Sepiolite is a 2:1 type nanofibrous clay containing structural blocks which alternate with structural cavities called as tunnels and channels. The structural blocks are composed of one central octahedral sheet and two tetrahedral silica sheets. The silanol groups $(\mathrm{Si}-\mathrm{OH})$ are exposed at the marginal surface of nanofibers due to the periodic upside down inversion of tetrahedral sheets [12, 13]. These silanol groups are easily accessible to numerous organic coupling agents for surface functionalization $[14,15]$. Recently vinyl functionalized 
sepiolite has been used as a promising substrate to graft acrylonitrile on it $[16,17]$. Organic portion of the nanohybrids are commonly grafted on inorganic part by polymerization of desired monomers.

The objective of this work is to report the comparative study on the synthesis of styrene grafted silanized sepiolite nanohybrids by simultaneous radiation grafting and emulsion graft polymerization. Both the techniques are compared by calculating grafting yield, gravimetrically. Absorbed radiation dose and monomer concentration is varied to study its effect on grafting yield. These CPNs can be used as ion exchange resins after sulfonation. Ion exchange resins have wide range applications in the fields of potable and industrial water treatment, water softening, nuclear reprocessing, metals removal and recovery and pharmaceutical technology.

\section{Experimental}

\subsection{Materials and Chemicals}

Sepiolite (SP), styrene (St), vinyltriethoxysilane (VTES), acetone and $\mathrm{HCl}$ were purchased from Sigma Aldrich. potassium persulphate (PPS) from Merck, Polysorbate 80 from Riedel-deHaen, Isopropanol from Daejung, teterahydrofuran (THF) from Lab-Scan. All these chemicals were of analytical grade.

\subsection{Synthesis}

\subsubsection{Silanization of Sepiolite}

To purify sepiolite from impurities, $10 \mathrm{~g}$ was mechanically stirred in $1 \mathrm{~L}$ water for $24 \mathrm{~h}$. After filteration, the dried SP was dispersed in isopropanol followed by the addition of hydrolyzed VTES $(108 \mathrm{mmol})$ drop wise and stirred at $60^{\circ} \mathrm{C}$ for 2 $\mathrm{h}$. Methanol was used to wash the mixture and then dried under vacuum. The silanized sepiolite (SS) was ground into fine powder [15].

\subsubsection{Simultaneaous Radiation Grafting}

Styrene (St) and SS (52 mmol of St /g of SS) were added in a pre-evacuated and pre-purged glass reactor. Under inert atmosphere, the grafting mixture was stirred for $6 \mathrm{~h}$ to form a stable suspension. Then it was irradiated in a cobalt-60 gamma cell $(24 \mathrm{kCi})$ at different absorbed doses with dose rate of $5 \mathrm{kGy} / \mathrm{hr}$ at room temperature under inert atmosphere. The resulting nanohybrids were washed with acetone followed by Soxhlet extraction for 7-8 $\mathrm{h}$ with THF to eradicate homopolymer. The grafted samples (SPS-R) were desiccated under vacuum until their weight became constant. Following formula was used to calculate grafting yield (GY, \%)

$$
G Y(\%)=\frac{W_{f}-W_{i}}{W_{i}} \times 100
$$

where $W_{i}$ and $W_{f}$ are the weights of SS and SPS-R, before and after grafting respectively. To study the effect of absorbed dose, different formulations were prepared for the synthesis of grafted nanohybrids which are presented in Table 1.

Table 1: Experimental formulations for the synthesis of SPS-R nanohybrids.

\begin{tabular}{|l|c|c|c|c|c|}
\hline Codes & SPS-R40 & SPS-R50 & SPS-R60 & SPS-R70 & SPS-R80 \\
\hline Sepiolite (g) & 1 & 1 & 1 & 1 & 1 \\
\hline Styrene (mmol) & 52 & 52 & 52 & 52 & 52 \\
\hline Absorbed dose (kGy) & 40 & 50 & 60 & 70 & 80 \\
\hline
\end{tabular}

\subsubsection{Emulsion Graft Polymerization}

Silanized sepiolite (SS), polysorbate 80 (surfactant) and water $(100 \mathrm{~mL})$ were charged in a $250 \mathrm{~mL}$ three-neck flask fixed with condenser. The reaction mixture was agitated moderately under inert atmosphere. Styrene monomer was added to the reaction mixture and heating was started. As the temperature reached to $70^{\circ} \mathrm{C}$, PPS (initiator) was introduced. After 4 $\mathrm{h}$ of the reaction, unreacted monomer was wiped out by washing the resulting nanohybrid with water and acetone. The homopolymer was removed with the same procedure as is mentioned in simultaneous radiation grafting method. The 
developed samples (SPS-E) were dried under vacuum at $60^{\circ} \mathrm{C}$ until completely dehydrated. Grafting yield (GY \%) was determined by means of eq. (1). Different preparations were made to learn the behaviour of monomer quantity on the production of nanohybrids which are presented in Table 2.

Table 2: Experimental formulations for the synthesis of SPS-E nanohybrids.

\begin{tabular}{|l|c|c|c|c|}
\hline Codes & SPS-E1 & SPS-E3 & SPS-E5 & SPS-E7 \\
\hline Styrene, \% (w/v) & 1.0 & 3.0 & 5.0 & 7.0 \\
\hline
\end{tabular}

\subsection{Characterization}

FT-IR spectrophotometer (Nicolet 6700, Thermo Electron Corporation) was used to get spectra of the developed nanohybrid materials. All the materials were scanned from 4000 to $500 \mathrm{~cm}^{-1}$ at the resolution of $6 \mathrm{~cm}^{-1}$. To attain high signal to noise ratio, 100 scans per sample were recorded. XRD analysis of the prepared samples was conducted with D8

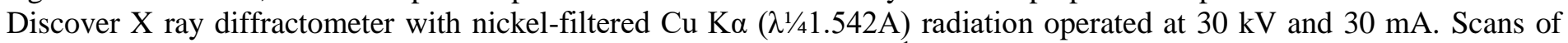
data were attained from $2 \theta=6^{\circ}-80^{\circ}$ at the scanning rate of $1.1^{\circ} \mathrm{min}^{-1}$. Tescan, MIRA-3 field emission scanning electron microscope (FESEM) joined with EDX detector was used to explore the alterations in the surface morphology of the developed samples. Before SEM analysis, the nanohybrids were coated with amorphous carbon to get conductive surfaces. Transmission electron microscope, Jeol JEM-2000FXII activated at $190 \mathrm{kV}$ with tungsten filament was utilized to examine the internal morphology. To determine the fiber diameter and length of sepiolite in SEM and TEM micrographs, Image $\mathbf{J}$ software was used.

\section{Results and Discussion}

The chemical reactions followed during the preparation of polystyrene nanohybrid by radiation grafting and emulsion graft polymerization is described in Fig.1

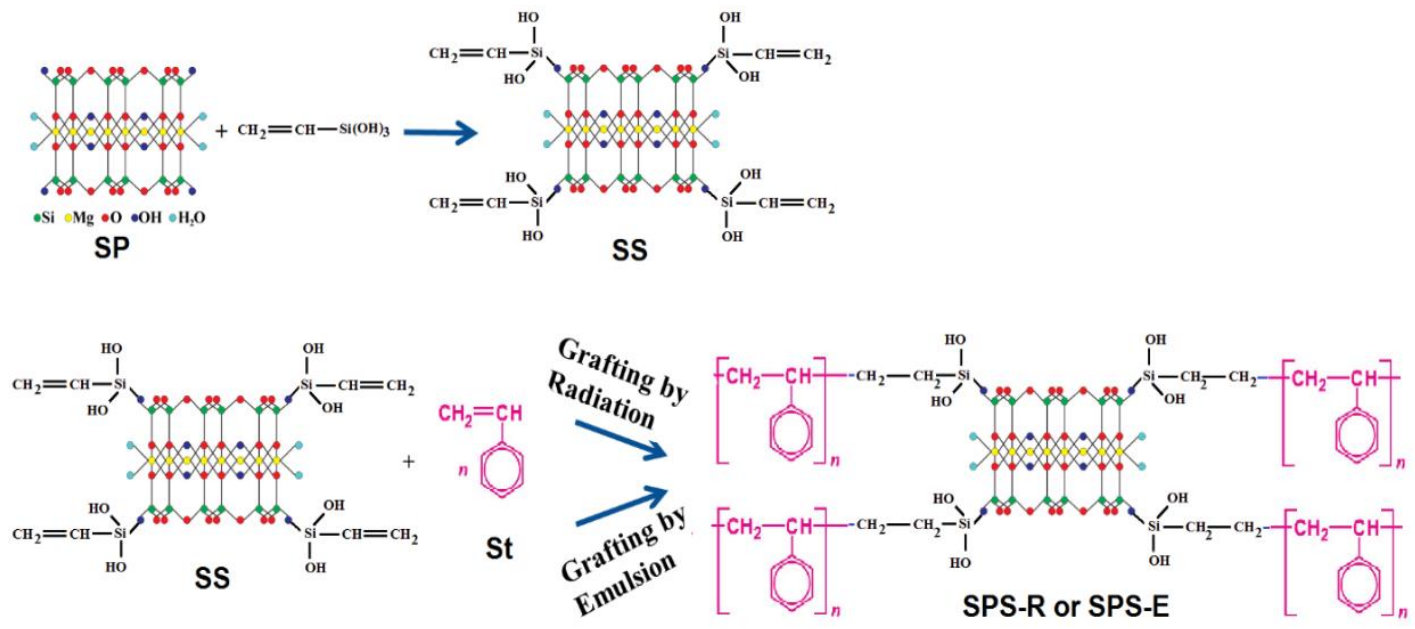

Fig. 1: Steps involved in the preparation of polystyrene nanohybrid material.

\subsection{Impact of Monomer Concentration and Absorbed Dose (Simultaneous Radiation Grafting)}

The outcome of smonomer amount on grafting degree of SPS-R is presented in Fig. 2A. The GY (grafting yield) has shown its direct relationship with St concentration and displayed its maximum grafting of $257 \%$ at $52.0 \mathrm{mmol} \mathrm{St} / \mathrm{g}$ of SS at the dose of $60 \mathrm{kGy}$. On further rise in St concentration, lowering of GY was observed. By enhancing the amount of monomer, the formation of homopolymer had shown increasing trend that eventually lessens the monomer availability to growing grafted chains.

The relationship between absorbed dose and GY is revealed in Fig. 2B. It is observed in this figure that grafting yield was augmented with the increase in absorbed dose. At $60 \mathrm{kGy}$, maximum grafting yield of $257 \%$ was attained and then it 
levelled off that might be due to the formation of homopolymer as a result of the reaction of primary radicals of monomers with each other. Homopolymer production increases the viscosity of the system thus hinders the approach of monomer radicals to the grafting chain on sepolite

\subsection{Impact of Monomer Concentration (Emulsion Graft Polymerization)}

The results of the influence of monomer concentration on grafting yield of SPS-E are presented in Fig. 2C. Low grafting yield can be observed with $1 \%$ St concentration. This describes that the amount of monomer is not enough for grafting on the substrate that conceivably holds more active sites available for grafting. By increasing the monomer amount to $3 \%$, maximum grafting of $85 \%$ was achieved. Above this concentration, the grafting yield was reduced that might be due to the formation of homopolymer which restricts the arrival of monomer molecules and its oligomers to the substrate.
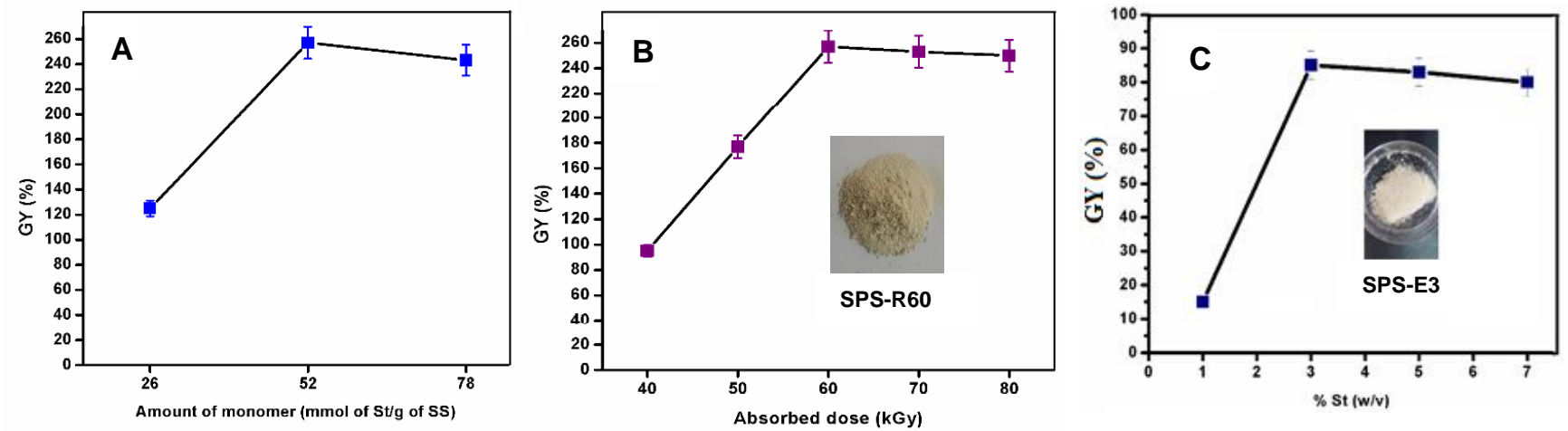

Fig. 2: (A) Impact of St concentration on grafting yield (GY, \%). (B) Influence of absorbed dose on grafting (52 mmol of St/g of SS, dose rate $=5.0 \mathrm{kGy} / \mathrm{h}$ ) with photograph of SPS-R60. (C) Effect of St amount on grafting yield with photograph of SPS-E3.

\subsection{FT IR Analysis}

Fig 3 is presenting the FT IR spectra of pure sepiolite and prepared nanohybrids. In Fig. 3A, band extending from $3690 \mathrm{~cm}^{-1}$ to $3417 \mathrm{~cm}^{-1}$ are representing the stretching (symmetric and asymmetric) of $\mathrm{OH}$ groups and vibration at 1660 $\mathrm{cm}^{-1}$ is representing $\mathrm{OH}$ bending vibrations. Vibrations towards the lower wavenumbers are indicating Si-O-Si and Si-O$\mathrm{Mg}$ linkages [15].

Fig. 3B (SS) is displaying additional vibrations at 2971 and $2886 \mathrm{~cm}^{-1}$ that are credited to $\mathrm{C}-\mathrm{H}$ stretching (asymmetric and symmetric) whereas $1391 \mathrm{~cm}^{-1}$ and $1277 \mathrm{~cm}^{-1}$ are ascribed to $\mathrm{C}-\mathrm{H}$ bending peaks. The increase in the intensity of 1660 vibration is accounted for the collective response of zeolitic water $(\mathrm{OH})$ and $\mathrm{C}=\mathrm{C}$ stretch. The band at $3631 \mathrm{~cm}^{-1}$ allocated to structural $\mathrm{OH}$, wiped out in SS spectrum which indicates the combination of $\mathrm{OH}$ groups of sepiolite (silanol groups) with VTES [18].

The presence of polystyrene grafts (aromatic rings) in the FT-IR spectrum of SPS-R60 (Fig. 3C) was recognised by the vibration of $=\mathrm{C}-\mathrm{H}$ stretch at $3051 \mathrm{~cm}^{-1}$. The existence of aromatic $\mathrm{C}=\mathrm{C}$ bond of aromatic rings in the grafted chains was indicated by supplementary vibrations at 1600,1583 and $1425 \mathrm{~cm}^{-1}$. The C-H symmetric and asymmetric stretching vibrations were characterised by vibrations at 2900-2800 and 3000-2900 $\mathrm{cm}^{-1}$ respectively. Mono substitution was established by presence of 750 and $690 \mathrm{~cm}^{-1}$ vibrations which represent aromatic out of plane $\mathrm{C}-\mathrm{H}$ deformation bands. The features of the SPS-R60 spectrum support the efficacious grafting of the polystyrene chains onto the silanized sepiolite. The decrease in 1660 vibration was a sign of the polymerization of styrene monomers through vinylic double bonds. Fig. 3D is representing the FT-IR spectrum of SPS-E3 which indicates the presence of specific bands for benzene ring with weaker intensity as compared to SPS-R60 (Fig. 3C). Comparison of SPS-R60 and SPS-E3 spectra is in agreement with grafting yield results. 

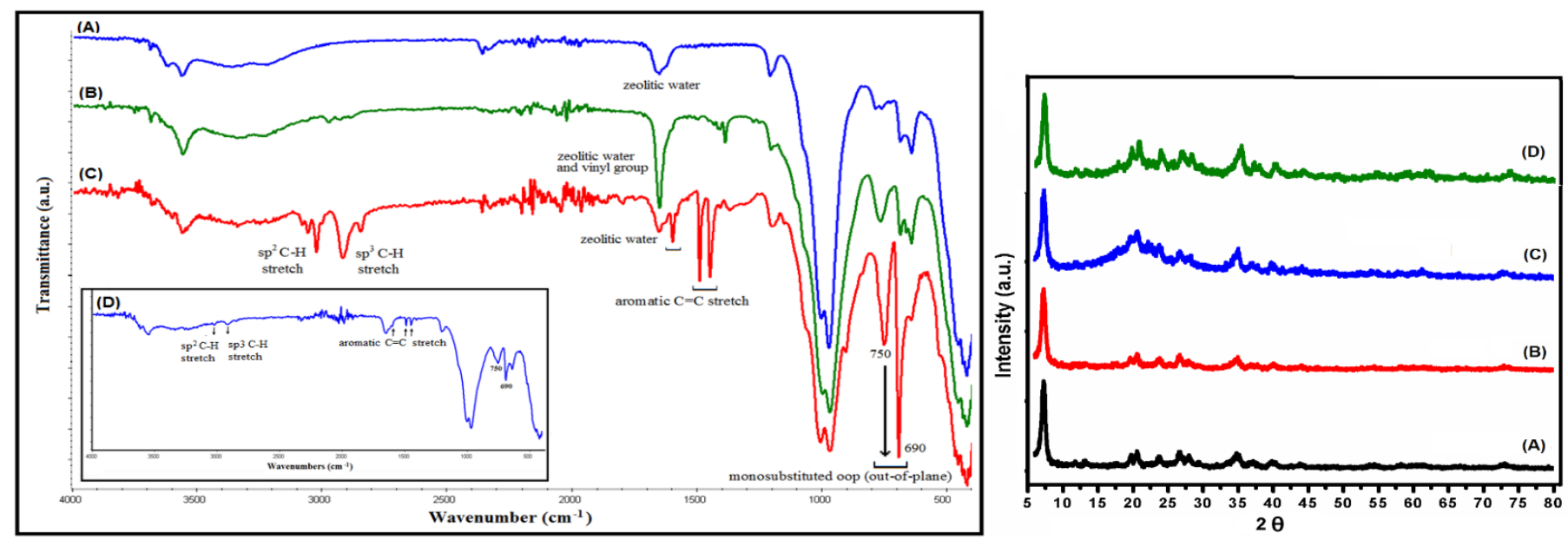

Fig. 3: FT-IR spectra and XRD diffractrograms of (A) SP, (B) SS, (C) SPS-R60, (D) SPS-E3.

\subsection{XRD Analysis}

Fig. 3 (right side) is displaying the XRD diffractograms of SP and its developed forms. The high intensity peak of SP was observed at $2 \theta=7.37^{\circ}(110)$. This distinctive peak of SP preserved its $2 \theta$ position in the developed forms. This indicated that the structural integrity of the clay was retained during the silanization and grafting stages. A slight reduction in the peak height and crystallinity in SS, revealed the combination of VTES at the peripheral surface of SP [15, 19]. The amorphous hump in SPS-R60 nanohybrid (Fig. 3C), is ascribed to polystyrene at $11^{\circ}$ and $17-26^{\circ}$ [20]. In SPS-E3 (Fig. 3D), the amorphous halo is comparatively smaller (comparison with Fig. 3C). XRD results are agreeing with the outcomes of FT-IR spectroscopy. The crystallinity $(\mathrm{X}, \%)$ of the developed samples was calculated by the formula in eq. 2 and are displayed in Table 3 [21].

$$
X(\%)=[\{\text { Area }(\text { total })-\text { Area }(\text { amorphous region })\} / \text { Area }(\text { total })] \times 100
$$

By studying the XRD diffractograms and crystallinity (X, \%), it was found that the crystallinity of SP was progressively reduced by the combination of amorphous polystyrene grafts with the crystalline substrate. The fall of crystallinity is greater in SPS-R60 as compared to SPS-E3. These interpretations emphasize towards the higher grafting yield of the developed nanohybrids by radiation grafting as compared to emulsion graft polymerization.

Table 3: Crystallinity (\%) of SP, SS, SPS-R60 and SPS-E3.

\begin{tabular}{|l|c|c|c|c|}
\hline Codes & SP & SS & SPS-R60 & SPS-E3 \\
\hline $\mathrm{X}(\%)$ & 83.34 & 80.11 & 35.82 & 68.74 \\
\hline
\end{tabular}

\subsection{Morphological Studies (FESEM and TEM)}

To explore the modifications in surface morphology of SP and its synthesized nanohybrids, FESEM attached with EDX detector was used to take the micrographs which are displayed in Fig. 4. SP exhibited its fibrous nature showing smoothness of the nanofibres surface. These nanofibers are agglomerated with each other. After silanization (SS), the agglomerated nanofibres were fragmented into short fibres with uneven surface which indicates the surface modification $[19,22]$. The SPS-R60 micrograph is displaying that all the nanofibers were wrapped up by polystyrene grafts. This figure was in accordance with the results of XRD since the structural veracity of SP was retained in SS and SPS-R60 because changes happened mainly on the external surface or by fractional substitution of zeolitic water [8]. Dense wrapping of nanofibers by polystyrene grafts is also supporting the high grafting yield. EDX spectra are displaying the results accordingly. In the SS micrograph, the diameter of the fibers was 30-45 nm and after emulsion graft polymerization, the fiber diameter was enlarged to 60-90 nm in SPS-E3. This micrograph is exhibiting the wrapping of SS nanofibers by polystyrene chains and grafted nanofibers are existing individually which supports the low grafting yield by emulsion grafting method. 


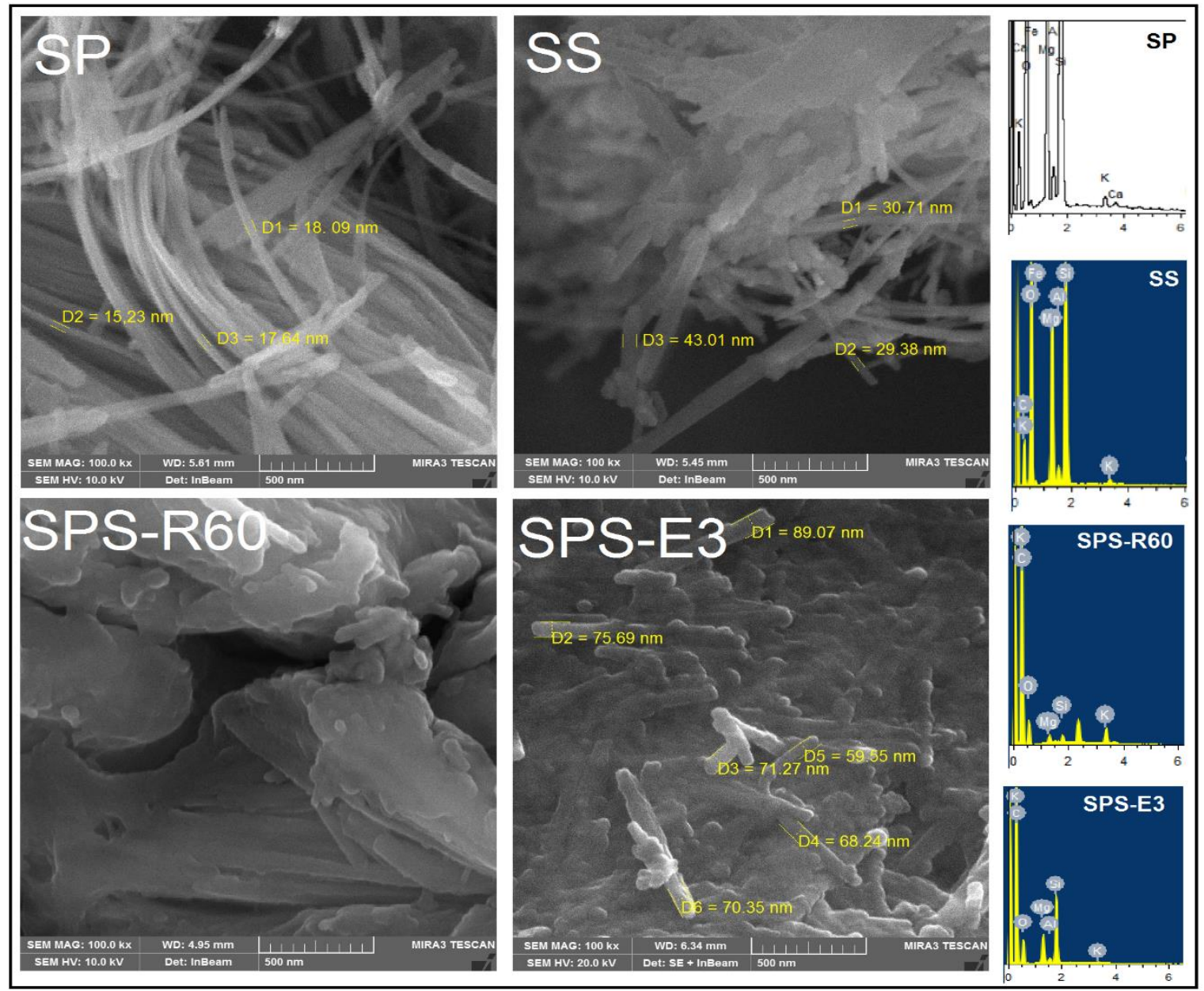

Fig. 4: FESEM micrographs of (A) SP, (B) SS, (C) SPS-R60, (D) SPS-E3 with their respective EDX spectra.

Emulsion graft polymerization mechanism, interpreted by the theories proposed by Harkins, Smith and Ewart [23,24], is displayed in Fig. 5 with TEM micrographs of SP and SS. Due to the hydrophobicity of styrene $(0.03 \%)$, it is generally accumulated in monomer droplet and it is negligibly present in the aqueous phase. The short nanofibers of SS which entered in the micelles, have gone through heterogeneous nucleation to form polystyrene grafted nanohybrids. Lengthy nanofibers stayed in the aqueous phase where no monomer molecule was found due to their hydrophobic nature. Thus homogeneous nucleation insignificantly occurred in water [25]. As illustrated by TEM micrograph of SS, during silanization of sepiolite, the nanofiber length was shortened to $5-500 \mathrm{~nm}$ approximately. These short nanofibers could simply move in the micelles where they grafted with styrene molecules and its oligomers. Conversely, due to the limited access of SS long nanofibers to St monomer in aqueous medium, they were unable to graft with each other. Resultantly, nanohybrids with lower grafting yield of St monomer by emulsion graft polymerization were attained. 

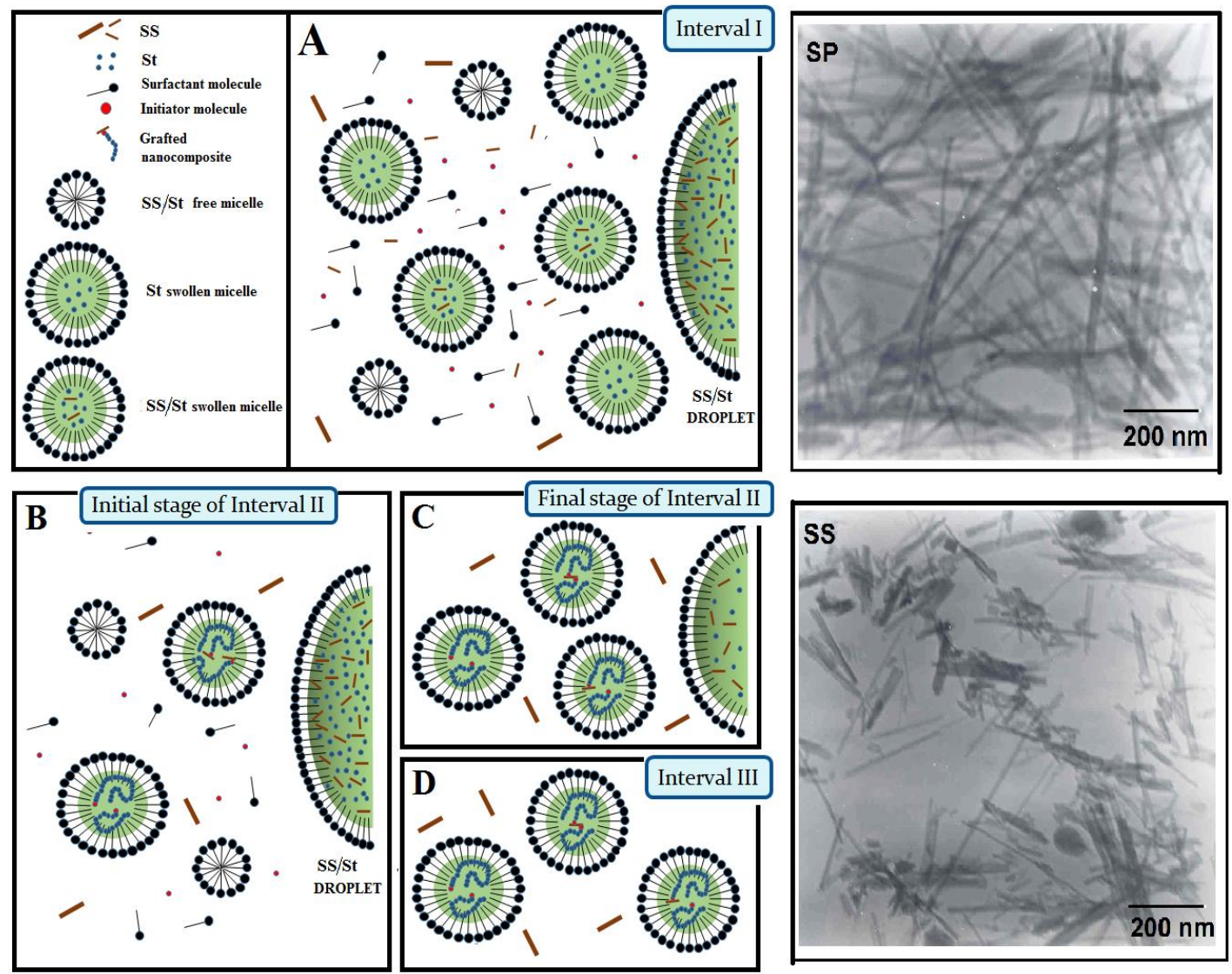

Fig. 5: Illustration of emulsion graft polymerization of styrene monomer (three intervals) with TEM micrographs of SP and SS.

\section{Conclusion}

Polystyrene grafted nanohybrids were successfully prepared by simultaneous radiation grafting and emulsion graft polymerization. The method of simultaneous radiation grafting was verified to be significantly influential since by exposing SS and styrene for $60 \mathrm{kGy}$ of absorbed dose, GY of $257 \%$ was attained. The dosage of $60 \mathrm{kGy}$ for $52 \mathrm{mmol}$ of St in $1 \mathrm{~g}$ of SS at ambient temperature under inert medium were validated to be the optimum grafting parameters. The grafting of PS chains on silanized sepiolite was confirmed by FT-IR, XRD and FESEM techniques. The outcomes of the above stated characterization methods recommend that St grafted nanohybrids have been developed by using gamma source.

PS grafted nanohybrids developed by emulsion graft polymerization have revealed comparatively lower grafting of merely $85 \%$ with $3 \% \mathrm{St}(\mathrm{w} / \mathrm{v})$. The occurrence of only heterogeneous nucleation in micelles resulted in low grafting yield as homogeneous nucleation did not take place in aquatic phase due to the negligible miscibility of styrene in water. FT-IR and XRD analyses are also in agreement with the gravimetric outcomes. The SEM micrographs are demonstrating the grafting progression as the diameter of SS nanofibers was improved after grafting but grafting yield is much lower (85\%) as compared to radiation grafting $(257 \%)$. It can be concluded from the above results and discussion that suitable and effective method for styrene grafting is simultaneous radiation graft polymerization as compared to emulsion graft polymerization.

\section{Acknowledgements}

The authors offer their gratitude to Nuclear Institute of Food and Agriculture (NIFA), Peshawar for providing facilities and permission to use gamma irradiator. 


\section{References}

[1] A. S. Z. Naseri and A. Jalali-Arani, "A comparison between the effects of gamma radiation and sulfur cure system on the microstructure and crosslink network of (styrene butadiene rubber/ethylene propylene diene monomer) blends in presence of nanoclay," Rad. Phys. Chem., vol. 115, pp. 68-74, 2015.

[2] T. Yamaki, M. Asano, Y. Maekawa, Y. Morita, T. Suwa, and J. Chen, "Radiation grafting of styrene into crosslinked PTEE films and subsequent sulfonation for fuel cell applications," Rad. Phys. Chem., vol. 67, pp. 403-407, 2003.

[3] A. Vahdat, H. Bahrami, N. Ansari, and F. Ziaie, "Radiation grafting of styrene onto polypropylene fibres by a 10 MeV electron beam," Rad. Phys. Chem., vol. 76, pp. 787-793, 2007.

[4] J. P. Rao and K. E. Geckeler, "Polymer nanoparticles: Preparation techniques and size-control parameters," Prog. Polym. Sci., vol. 36, pp. 887-913, 2011.

[5] A. Aerdts, S. J. C. Theelen, T. M. C. Smit, and A. L. German, "Grafting of styrene and methyl methacrylate concurrently onto polybutadiene in semicontinuous emulsion processes and determination of copolymer microstructure," Polymer, vol. 35, pp. 1648-1653.

[6] Z. Sedlakova, J. Plestil, J. Baldrian, M. Slouf, and P. Holub, "Polymer-clay nanocomposites prepared via in situ emulsion polymerization," Polym. Bull., vol. 63, pp. 365-384, 2009.

[7] T. A. Elbokl and C. Detellier, "Aluminosilicate nanohybrid materials. Intercalation of polystyrene in kaolinite," $J$. Phys. Chem. Solids, vol. 67, pp. 950-955, 2006.

[8] M. Alshabanat, A. Al-Arrash, and W. Mekhamer, "Polystyrene/montmorillonite nanocomposites: Study of the morphology and effects of sonication time on thermal stability," J. nanomater., vol. 2013, pp. 1-12, 2013.

[9] P. Gupta, M. Bera, and P. K. Maji, "Nanotailoring of sepiolite clay with poly [styrene-b-(ethylene-co-butylene)-bstyrene]: structure-property correlation," Polym. adv. technol., vol. 28, pp. 1428-1437, 2017.

[10] H. Roghani-Mamaqani, V. Haddadi-Asl, M. Najafi, and M. Salami-Kalajahi, "Preparation of tailor-made polystyrene nanocomposite with mixed clay-anchored and free chains via atom transfer radical polymerization," AIChE J., vol. 57, pp. 1873-1881, 2011.

[11] Y. Zhang, Q. Liu, S. Zhang, Y. Zhang, Y. Zhang, and P. Liang, "Characterization of kaolinite/styrene butadiene rubber composite: Mechanical properties and thermal stability," Appl. Clay Sci., vol. 124-125, pp. 167-174, 2016.

[12] E. Ruiz-Hitzky, M. Darder, F. M. Fernandes, B. Wicklein, A. C. S. Alcantara, and P. Aranda, "Fibrous clays based bionanocomposites," Prog. Polym. Sci. , vol. 38, pp. 1392-1414, 2013.

[13] E. Galan, "Properties and applications of palygorskite-sepiolite clays," Clay Miner., vol. 31, pp. 443-453, 1996.

[14] D. G. Lopez, J. F. Fernandez, J. C. Merino, J. Santaren, and J. M. Pastor, "Effect of organic modification of sepiolite for PA 6 polymer/organoclay nanocomposites," Compos. Sci. Technol., vol. 70, pp. 1429-1436, 2010.

[15] J. Di, L. Jin-Quan, and Y. Xing-Fang, "Modification of sepiolite by treatment with methyl triethoxysilane," Mater. Sci., vol. 19, pp. 44-47, 2004.

[16] S. Taimur and T. Yasin, "Influence of the synthesis parameters on the properties of amidoxime grafted sepiolite nanocomposites," Appl.Surf. Sci., vol. 422, pp. 239-246, 2017.

[17] S. Taimur, M. I. Hassan, and T. Yasin, "Removal of copper using novel amidoxime based chelating nanohybrid adsorbent," Eur. Polym. J., vol. 95, pp. 93-104, 2017.

[18] M. Shafiq, T. Yasin, and S. Saeed, "Synthesis and characterization of linear low-density polyethylene/sepiolite nanocomposites," J. Appl. Polym. Sci., vol. 123, pp. 1718-1723, 2012.

[19] X. Liang, Y. Xu, and G. Sun, "Preparation and characterization of mercapto functionalized sepiolite and their application for sorption of lead and cadmium," Chem, Eng. J., vol. 174, pp. 436-444, 2011.

[20] S. Hasegawa, K. Sato, T. Narita, Y. Suzuki, S. Takahashi, N. Morishita, and Y. Maekawa, "Radiation-induced graft polymerization of styrene into a poly(ether ether ketone) film for preparation of polymer electrolyte membranes," $J$. Membr. Sci., vol. 345, pp. 74-80, 2009.

[21] M. Shafiq, M. S. Mehmood, and T. Yasin, "On the structural and physicochemical properties of gamma irradiated UHMWPE/silane hybrid," Mater. Chem. Phys., vol. 143, pp. 425-433, 2013.

[22] N. Garcia, J. Guzm, and E. Benito, "Surface modification of sepiolite in aqueous gels by using methoxysilanes and its impact on the nanofiber dispersion ability," Langmuir, vol. 27, pp. 3952-3959, 2011. 
[23] J. L. Gardon, "Emulsion polymerization I. Recalculation and extension of Smith- Ewart Theory," J. Polym. Sci. A., vol. 6, pp. 623-641, 1968

[24] C. S. Chern, "Emulsion polymerization mechanisms and kinetics," Prog. Polym. Sci., vol. 31, pp. 443-486, 2006.

[25] H. B. Yamak, "Emulsion polymerization: Effects of polymerization variables on the properties of vinyl acetate based emulsion polymers," in Polymer Science, pp. 35-72, 2013. 